Annals of Plant Sciences

ISSN: 2287-688X

OPEN ACCESS

Research Article

www.annalsofplantsciences.com

\title{
Check list of diatom taxa from Vindhyan rivers in Central Highland region
}

Jyoti Verma $^{1 *}$, Prateek Srivastava ${ }^{2}$, Ambrina Sardar Khan ${ }^{2}$, Anita Gopesh ${ }^{1}$

1Department of Zoology, University of Allahabad, Allahabad 211002, Uttar Pradesh, India.

2Amity Institute of Environmental Sciences, Amity University, Noida 201 303, India.

Received: 9/13/2017; Accepted: 9/23/2017

Abstract: Investigations were undertaken for the first time from the freshwater epilithic diatoms of the Vindhyan Rivers (Ken, Tons, Paisuni), Central Highland, India. Epilithic samples (33 nos) were obtained by scraping an area of $3 \times 3 \mathrm{~cm}$ from cobbles at the 11 sampling stations (Ken, Tons, Paisuni) situated between latitude $23^{\circ} 30^{\prime}$ to $26^{\circ} \mathrm{N}$ and longitude $78^{\circ} 30^{\prime}$ to $82^{\circ} 30^{\prime} \mathrm{E}$. In all 293 diatom taxa (species, varieties and forms) belonging 49 genera were identified, along with some unidentified forms.

Keywords: Ken, Tons, Paisuni, Navicula, Niťsschia

\section{Introduction}

No investigations exist on the diatom flora of Vindhya region in Central Highlands. There have been few studies in the Indo-Gangetic Plains from Varanasi and Allahabad (Singh 1961, 1962, 1963; Chadha 1979; Pandey \& Pandey 1980 a, b, c, 1983; Pandey et al., 1983; Gupta \& Agrawal 2007; Nautiyal et al., 2010). Hence, studies were made to generate information on the diatom flora of the Vindhya region. The present contribution provides a check list of diatom taxa from the Central highland region, Vindhya in particular, which has not been explored earlier.

\section{Study-area}

The rivers selected in Vindhya were located between $23 \circ 30^{\prime}$ to $26^{\circ} \mathrm{N}, 78^{\circ} 30^{\prime}$ to $82^{\circ} 30^{\prime} \mathrm{E}$. The Vindhya rivers Ken, Paisuni and Tons flow north from low (north of Narmada, around Tropic of Cancer) to high latitude along southern fringe of the Indo-Gangetic Plains. The Ken and Paisuni are right-bank tributaries of the Yamuna River, while Tons that of the Ganga. Ken $(340 \mathrm{~km})$ and Tons $(305 \mathrm{~km})$ were relatively larger drainage compared to the Paisuni $(100 \mathrm{~km})$ having an average gradient of $0.91 \mathrm{~m} \mathrm{~km}^{-1}, 2.0 \mathrm{~m} \mathrm{~km}^{-1}$ and $1.02 \mathrm{~m} \mathrm{~km}^{-1}$, respectively. Except for the mouth zone these rivers vary topographically.

\section{Materials and Methods}

Sampling: Collections were made from 20 stations; 11 stations on 3 rivers in the Vindhya region. Sampling was performed across the width of the stream at the depth of $15-30 \mathrm{~cm}$ along the accessible banks. Diatoms were collected by scraping surface of stony substrate and preserved in formalin. These samples were first cleaned with double distilled water to remove traces of formaldehyde. Samples were given Hydrochloric acidperoxide treatment, washed repeatedly and mounted in Naphrax. Each slide was examined by UPLANAPO x100 oil immersion objective under bright field to identify the species. Images of each taxon were obtained by NIKON Digital Imaging System and processed with Adobe Photoshop to prepare the photographic plates. Identifications were made according to standard literature (Verma 20011). The permanent mounts have been adequately stored at the Aquatic Biodiversity Unit,

\section{${ }^{*}$ Corresponding Author:}

\section{Dr. Jyoti Verma,}

Department of Zoology, University of Allahabad,

Allahabad, Uttar Pradesh, India

E-mail: diatombuster@gmail.com
Department of Zoology, H. N. B. Garhwal University, Srinagar.

\section{Check list}

The Suborder Coscinodiscineae is represented by Family Thalassiosiraceae occurred as Aulacoseira (1 species) and Cyclotella (2 species).

\section{Aulacoseira}

1. Aulacoseira granulata (Ehrenb.) Simonsen. Metzeltin et al., 2005, Iconographia Diatomologica, p. 1, figs. 1-4

\section{Cyclotella}

2. Cyclotella menegbiniana Kütz., Hustedt 1930, p. 100, fig. 67a. 3. C. psendostelligera Hustedt 1939, p. 581, figs 1, 2.

The Suborder Araphidineae is represented by Fragilariaceae. This family is represented by genera Diatoma (4 species), Fragilaria (2 species), Staurosira (2 species), Synedra (19 species) and Tabellaria (1species).

\section{Diatoma}

4. Diatoma mesodon (Ehrenb.) Kütz. 1844, p. 47, pl. 17: fig. XIII.

5. D. minus Grun., Schmidt 1902, p. 268, figs. 60-61.

6. D. temuis (Agardh) Van Heurck Synopsis 1885, p. 160.

7. D. vulgare var. productum (producta) Grun. 1862, p. 49 fig. 363.

\section{Fragilaria}

8. Fragilaria cf. capucina Desmaziers, Metzeltin et al., 2005, p. 14, figs. 26-27.

9. F. capucina var. vaucheria (Kütz.) Lange-Bert., 1980, p. fig. 747 .

\section{Staurosira}

10. Staurosira longirostris (Frenguelli) Metzeltin et al., 2005, p. 13 figs. 4-13.

11. S. pinnata Ehrenb. 1843, 1. 3, figs 6, 8

\section{Synedra}

12. Synedra acus Kütz., 1844, p. 68, pl. 15: fig. 7 .

13. S. acus var. angustissima (Grun.) van Heurck 1885, p. 151

14. S. acus var. radians (Kütz.) Hustedt 1930, p. 155

15. S. acus var. 1 Kütz., 1844, p. 68, pl. 15: fig. 7.

16. S. amphicephela Kütz.,1844, p. 64, pl. 3: fig. 12.

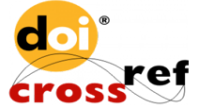


17. S. capitata Ehrenb. 1836, p. 53

18. S. dorsiventralis O.Müll., 1910, p. 45, pl. 114, fig. 3.

19. S. rumpens Kütz. 1844 , p. 69 , pl. 16/6, figs. $4-5$

20. S. rumpens var. familiaris (Kütz.)Hustedt1930,p.40, figs.15, 16.

21. S. rumpens var. fragilaroides Grun., Hustedt 1930, fig.12.

22. S. tabulata (C. Agardh) Kütz. 1844, p. 68, pl. 15, fig. 10

23. S. tabulata var. fasciculata (Kütz.) Grun.,Hustedt 1985,fig. 15.

24. S. ulna (Nitzsch) Ehrenb.1832, p. 87.

25. S. ulna var. aequalis (Kütz.) Hustedt, 1914, p. 45.

26. S. ulna var. amphirbynchus (Ehrenb.) Grun.1862, p.83,fig. 397

27. S. ulna var. biceps (Kütz.) Schönfeldt 1913, fig. 39.

28. S. una var. danica (Kütz.) Grun., p. 151; pl. 38, fig.14A

29. S. ulna var. spathulifera Grun., pl. 38, fig. 4.

30. Synedra sp 1. Ehren., 1830.

\section{Tabellaria}

31. Tabellaria flocculosa (Roth) Kütz. 1844, p. 17, fig. 21.

The Suborder Raphidineae is represented by family Eunotiaceae, Achnanthaceae, Naviculaceae, Epithemiaceae, Bacillariaceae and Surirellaceae. Eunotiaceae Kütz. 1844 is represented by genus Eunotia (6 species) only.

\section{Eunotia}

32. Eunotia sp.1 Ehren., 1837.

33. Eunotia sp.2 Ehren., 1837.

34. Eunotia lunaris (Ehrenb.) Grun. pl. 35, figs. 3,4,6.

35. E. pseudopectinalis Metzeltin et al., 2005, p. 24, figs. 15-18.

36. E. pseudofaba Metzeltin et al., 2005, p. 18, figs. 9-12.

37. E. sudetica O. Muller, Hustedt 1914, p. 55.

Family Achnanthaceae Kütz. 1844 is represented by genera Achnanthes (1 species), Achnanthidium (14 species), Planathodium (5 species) and Cocconeis (6 species).

\section{Achnanthes}

38. Achnanthes coarctata (Brébisson) Grun. Cleve \& Grun. 1880, p. 20.

\section{Achnanthidium}

39. Achnanthidium biasolettiana Grun. var. subatomus LangeBertalot 1989, p. 28, pl. 57: fig. 8, pl. 59: figs. 1-11.

40. A. biasolettiana Grun. var subatomus Lange-Bertalot 1989, p. 28, pl. 57: fig. 8, pl. 59: figs. 1-11.

41. A. cf. exilis Kütz. 1833 , p. 577 ; pl. 16, fig. 53.

42. A. exigua Grun. var. exigua, K. Sv. Vet. Akad. Handl. 1880, vol. 17,2 , p. 21.

43. A. exigua var. constricta Torka Hustedt 1921, p.145, figs. 7, 8

44. A. linearis (W. Smith) Grun., Round \& Bukhtiyarova 1996, figs.37: 19-23 or Achnanthes petersenii Hustedt, 1936, p.179, figs 10-14.

45. A. minutissima var. gracillima (Meister) Lange-Bertalot, Round \& Bukhtiyarova 1996, figs. 54: 21-32, 55: 1-3

46. A. minutissima var. minutissima Kütz. 1833, p. 578, fig. 54.

47. A. minutissima var. minutissima Kütz. 1833 , p. 578 , figs. 54 (figs. 51: 1-20 u.a., vgl. REM-Figs.auf Tafel 52 u. 56) or var.?

48. A. minutissima var. jackii (Rabenhorst) Lange-Bertalot et Ruppel 1980, p. 18.

49. A. minutissima var. scotica (Carter) Lange-Bertalot in LangeBertalot \& Krammer 1989, Bibliothica Diatomologica, figs. 34: 1-6.

50. A. modestiformis Lange-Bertalot in Lange-Bertalot \& Krammer 1989, Bibliothica Diatomologica, figs. 55: 33-42'

51. A. petersenii Hustedt 1936, Bot.Arch. p.38, pl.179, figs 10-14.

52. A. taeniata Grun. 1880 , p. 22, pl. 1, fig. 5.

\section{Planothidium}

53. Planothidium lanceolata var. elliptica Cleve 1891,figs. 86: 33-34.

54. P. lanceolata (Brebisson) Grun. var. lanceolata, Lange-Bertalot 1999 , p. 281.

55. P. lanceolata fo. rostrata (Ǿstrup) Lange-Bertalot 1999, p.6, pl. 279.

56. P. lanceolata ssp. frequentissima Lange-Bertalot 1993,p.27,pl. 4.
57. P. lanceolata ssp. dubia (Grun.) Lange-Bertalot 1997, p.148, pl.206.

\section{Cocconeis}

58. Cocconeis pediculus Ehrenb. 1838, p. 194, pl. 21: fig. 11.

59. C. placentula Ehrenb. 1838, p. 194.

60. C. placentula var. euglypta (Ehrenb.) Cleve 1895, p. 170.

61. C. placentula var. lineata (Ehrenb.) Van Heurck 1885, p. 133 , pl.30, figs 31-32.

62. C. cf. scutellum Ehren. 1838, p. 194, pl. 14: fig. 18.

63. Cocconeis sp. Ehren., 1837, p. 173.

Family Naviculaceae Kütz. 1844 is represented by genera Amphipleura (1 species), Amphora (12 species), Anomoeoneis (1 species), Brachysira (2 species), Caloneis (6 species), Cymbella (31 species), Cymbopleura (14 species), Encyonema (5 species), Diploneis (6 species), Frustulia (1 species), Gomphocymbelopsis (1 species), Gomphonema (15 species), Gyrosigma (3 species), Mastogloia (1 species), Navicula (32 species), Navicula sensu lato (5 species), Adlafia (2 species), Aneumastus (2 species), Craticula (5 species), Diadesmis (2 species), Fallacia (2 species), Geissleria (1 species), Hippodonta (2 species), Luticola (8 species), Placoneis (2 species), Sellaphora (7 species), Neidium (4 species), Pinnularia (7 species), Rhoicosphenia (1 species), Scoliopleura (1 species) and Stauroneis (2 species).

\section{Amphipleura}

64. Amphipleura pellucida (Kütz.) Kütz. 1844, p. 103, pl.3, fig.52, pl.30, fig.8.

\section{Amphora}

65. Amphora aequalis Krammer 1980, p. 212-213, figs. 25-35.

66. A. calumetica (Thomas) Peragallo 1897, figs. 153: 8, 9 .

67. A. chaurra Metzeltin et al., 2005, p. 131 figs. 1-7.

68. A. copulata (Kütz.) Schoeman \& R.E.M. Archibald 1986, p. 429.

69. A. inariensis Krammer 1980, p.211, figs.21-24, 36-37, 43-45.

70. A. libyca Ehrenb 1841, p. 205.

71. A. montana Krasske 1932, p. 119 pl.2, fig.27.

72. A. normanii Rabenhorst 1864, p. 88.

73. A. ovalis (Kütz.) Kütz. 1844, p. 107.

74. A. pediculus (Kütz.) Grun. ex A.Schmidt 1875, pl.26: fig. 99.

75. A. twentiana Krammer 2003, figs. 138: 25-29.

76. A. veneta Kütz. 1844, p. 108, pl.3, fig.25.

\section{Anomoeoneis}

77. Anomoeoneis sphaerophora E.Pfitzer 1871, p. 77, pl.3, fig.10

\section{Brachyasira}

78. Brachysira vitrea (Grun.) R.Ross in Hartley 1986, Journal of the Marine Biological Association of the United Kingdom, p. 607.

79. Brachyasira sp. Kütz., 1836

\section{Caloneis}

80. Caloneis bacillum (Grun.) Cleve 1894, Le Diatomiste, p. 99.

81. C. cf. bacillum (Grun.) Cleve 1894, Le Diatomiste, p. 99

82. C. beccariana (Grun.) Cleve 1984, Synopsis of the naviculoid diatoms, p. 283, fig. 50.

83. C. silicula (Ehren.) Cleve 1894, Synopsis of the naviculoid diatoms, p. 51.

84. C. silicula var. elliptica Frengulli, Metzeltin et al., 2005, Iconographia Diatomologica, p. 155, figs. 17-22.

85. Caloneis sp. P.Cleve, 1894, p. 46.

\section{Cymbella}

86. Cymbella aspera (Ehren.) Cleve 1894, Synopsis of the naviculoid diatoms, p. 175

87. C. australica (A.Schmidt) Cleve 1894, Synopsis of the naviculoid diatoms, p. 176

88. C. bengalensis Grun. in A. Schmidt et al., 1875, Atlas der Diatomaceen-kunde pl. 9, fig.12,13 
89. C. bengaliformis Krammer 2002, Diatoms of Europe 3, p. 172, pl. 142: figs 8-9.

90. C. cymbiformis C.Agardh 1830, Conspectus Criticus Diatomacearum, p. 10

91. C. diversa Krammer 2002, Diatoms of Europe 3, p. 168, pl. 68: figs 1-9.

92. C. excisa Kütz. 1844, Die kieselschaligen Bacillarien oder Diatomeen, p. 80, pl.6, fig.17.

93. C. excisa var. angusta Krammer 2002, Diatoms of Europe 3, p. 159, pl. 9: figs 8-13.

94. C. excisa var. excisa Krammer 2002, figs. 5: 1, 8: 1-26, 9: 19 25, 12: 6.

95. C. excisa var. procera Krammer2002, p. 159, pl. 9: figs 1-7.

96. C. gracillifomis Krammer 2002, p. 167, pl. 54: figs 12-15.

97. C. hant:schiana Krammer 2002, figs. 27: 8-14; 28: 1-19; 29: 1-12; 30: 9-14

98. C. belvetica Kütz. 1844, p. 79, pl. 6: fig. 13.

99. C. kappii (Cholnoky) Cholnoky 1956, p.61, fig.17-20.

100. C. kolbei Hustedt 1949, p. 46; pl.1, fig.20-26.

101. C. kolbei var. kolbei Krammer 2002, figs. 14: 8-23: 31: 8, 9

102. C. lancetuliformis Krammer 2002, p. 167, pl. 54: figs 7-11.

103. C. metreltinii Krammer 2002, p. 166, pl. 49: figs 6-11.

104. C. neoleptoceros Krammer 2002, p. 134,173; pl.156, fig.1-8; pl.157, fig.1-19.

105. C. novazeelandiana Krammer 2002, p. 166, pl. 47: figs 6-9.

106. C. omaniana Krammer 2002, figs. 55: 1-6.

107. C. parva (W.Smith) Wolle 1890, pl. 7, fig. 19

108. C. parviformis Krammer 2002, figs. 15: 8-12 (2002).

109. C. pervarians Krammer 2002, p. 164, pl. 41: figs 7-12 (2002).

110. C. simonsenii Krammer in Krammer \& Lange-Bertalot 1985, pl.7, fig. $1-9$.

111. C. sumatrensis Hustedt 1937, p. 429, pl. 25: figs 17-19

112. C. tropica Krammer 2002, p. 164, pl. 44: figs 7-10.

113. C. tumida (Brébisson in Kütz.) van Heurck 1880, p. 64, pl.2, fig. 10 .

114. C. turgidula Grun. in A. Schmidt et al., 1875, pl.9,figs. 23-26.

115. C. turgidula var. venezoleiana Krammer 2002, p. 166, pl. 48 : figs $12-17$.

116. C. vulgata Krammer 2002, figs. 32: 7-13; 36: 1-14; 37: 16-21; 38: 1-18; 39: $1-7$

117. Cymbella sp. C.Agardh, 1830.

\section{Cymbopleura}

118. Cymbopleura angustata Krammer 2003, figs. 102: 1-7; 105: 917b; 109: 12-16.

119. C. angustata var. tenuis Krammer 2003, figs. 102: 8-10; 103 : 1-6.

120. C. citrus (Catrter and Bailey-Watts) Krammer 2003, figs. 97: 5-12.

121. C. falaisensis Grun., Krammer \& Lange-Bertalot 1985, p. 2 (1), figs.134: 14-22

122. C. kuelbsii Krammer 2003, figs. 113: 1-7b; 127: 11, 12, 19, 20-27.

123. C. kuelbsii var. nonfasciata Krammer 2003, figs. 127: 20-27.

124. C. lapponica Grun. ex Cleve 1894, p. 165, pl.4, fig.28.

125. C. naviculiformis (Auerswald) Krammer 2003, figs. 76 1-13; 771-13; 781-8;79;1-14; 8012; 821-12; 839-11.

126. C. rupicola (Grun.) Krammer 2003, figs. 66: 1-17; 67: 1-18.

127. C. rupicola sp.1 (Grun.) Krammer 2003, figs. 66: 1-17; 67: 118.

128. C. microcephala Grun. in van Heurck 1885, p. 63; pl.8, fig.3639

129. C. sparsistriata var. parva Krammer 2003, figs. 132: 21-33.

130. C. vrana Lange-Bertelot \& Krammer 2003, figs. 93: 9-18.

131. Cymbopleura sp. Krammer, 1999.

\section{Encyonema}

132. Encyonema jemtlandicum var. venezolanum Krammer 1997, p. 166, pl. 14: figs 1-5, pl. 50: fig. 8.

133. E. minutum (Hilse) D.G.Mann in Round, Crawford \& Mann 1990, p. 667.

134. E. perminutum Krammer Metzeltin et al., 2005, p. 130, figs. 6-15.

135. E. silesiacum (Bleisch) D.G.Mann 1990, figs. 117: 1-24.

136. E. vulgare Krammer var. vulgare Krammer 1997, Bibliothica Diatomologica 36, p. 167, pl. 36: figs. 4-10.

\section{Diploneis}

137. Diploneis minuta Petersen 1928, The aerial algae of Iceland, p. 381 , fig. 6.

138. D. oblongella (Nägeli) Cleve-Euler in Cleve-Euler \& Osvald 1922, Beschreibung zur Kartenblatter Väse, p. 57.

139. D. petersenii Hustedt 1937, Archiv für Hydrobiologie, figs. 110: 16-17.

140. D. pseudovalis Hustedt 1930, Bacillariophyta, p. 253, fig.403.

141. D. smithii (Brébisson) Cleve 1894, synopsis of the naviculoid diatoms, p. 96.

142. D. subovalis Cleve 1894, Planktonundersökningar, Cilicoflagellater och Diatomacéer, figs. 109: 8-9.

\section{Frustulia}

143. Frustulia weinholdii Hustedt 1937, Kryptogamen-Flora von Deutschland, pl.406, figs.7-8.

\section{Gomphonema}

144. Gomphonema angustum C.Agardh 1831, Conspectus Criticus Diatomacearum, p. 33.

145. G. angustatum (Kütz.) Rabenhorst 1864, Flora europaea algarum aquae dulcis et submarinae, p. 283.

146. G. augur var. spheriophorum (Ehrenb.) Grun. 1878, Algen und Diatomaceen aus dem Kaspischen Meere, fig. 157: 9.

147. G. clevei Fricke in Schmitd et al., 1902, Atlas der Diatomaceen-kunde, pl. 234: figs. 44-46.

148. G. angur var. turris (Ehrenb.) Lange- Bertalot, Hustedt $1937-$ 1938, Schlu_. Arch. Hydrobiol., Suppl. p. 439, pl. 28, figs. 14-16.

149. G. gracile Ehren. 1838, Infusionsthierchen als vollkommene Organismen 217; pl.18, fig.3.

150. G. gracile var. lanceolatum (Kütz.) Cleve 1894, Synopsis of the naviculoid diatoms, p. 183

151. G. lagenula Kütz. Metzeltin et al., 2005, Iconographia Diatomologica, p. 145, figs. 12-18.

152. G. lanceolatum Ehren., Kütz. 1844, Kieselagen Bacillarien oder Diatomeen, 87, pl. 29: fig. 73.

153. G. minutum (C.Agardh) C.Agardh 1831, Conspectus Criticus Diatomacearum, p. 34

154. G. parvulum (Kütz.) Kütz. 1849, Species algarum, p.65

155. G. parvulum var. micropus (Kütz.) Cleve, Schmidt 1902, Rep. Norweg. Fish. Invest., pl. 234, Figs. 16-17.

156. G. pumilum var. rigidum Reichardt \& Lange-Bertalot Metzeltin et al., 2005, Iconographia Diatomologica, p. 145, figs. 37-44.

157. G. sp. cf. apicatum Ehrenb., Metzeltin et al., 2005, Iconographia Diatomologica, p. 138, figs. 11-14.

158. G. truncatum Ehren. 1832, Abhandlungen der Königlichen Akademie Wissenschaften zu Berlin, p. 88.

159. Gomphonema sp. Ehren., 1832.

\section{Gomphocymbellopsis}

160. Gomphocymbellopsis ancyli (Cleve) Krammer 2003, Diatoms of Europe 4, 128; pl.143, fig.1-20; pl.144, fig.10-19; pl.145, fig.1-3.

\section{Gyrosigma}

161. Gyrosigma acuminatum (Kütz.) Rabenhorst 1853, Die Süssisser-Diatomaceen (Bacillarien.), p. 47; pl.5, fig.5a.

162. G. attenuatum (Kütz.) Cleve 1894, Synopsis of the naviculoid diatoms, p. 115

163. G. scalproides (Rabenhorst) Cleve 1894: 118118.

\section{Mastogloia}

164. Mastogloia smithii Thwaites in W. Smith (1856) Syn. Brit. Diat. p. 2, fig. 65.

\section{Navicula}

165. Navicula amphiceropsis Lange-Bertalot \& Rumrich in Rumrich, Lange-Bertalot \& Rumrich 2000, Diatoms of the Andes, p. 153; pl.42, figs.1-12.

166. N. angusta Grun. 1860, Über neue oder ungenügend gekannte Algen, p. 528; pl.3, fig.19.

167. N. antonii Lange-Bertalot in Rumrich et al., 2000, Annotated Diatoms Micrographs, p. 155. 
168. N. capitatoradiata Germain 1981, Proc. 6 ${ }^{\text {th }}$ International Diatom Symposium Budapest, p. 188; pl.72, fig.7.

169. N. cataracta-rheni Lange-Bertalot 1993, Bibliotheca Diatomologica, p. 99; pl.59, fig.13-15.

170. N. caterva Hohn \& Hellermann 1963, Transactions of the American Microscopical Society, p. 296; pl.3, fig.38.

171. N. cincta (Ehren.) Ralfs in Pritchard 1861, History of Infusoria, p. 901.

172. N. cryptocephala Kütz. 1844, Die kieselschaligen Bacillarien oder Diatomeen, p. 95, pl. 3: figs. 20, 26.

173. N. cryptofallax Lange-Bertalot \& Hofmann in LangeBertalot 1993, Bibliotheca Diatomologica, p. 103; pl.47, fig. 11; pl.48, figs.1-4.

174. N. cryptotenella Lange-Bertalot in Krammer \& LangeBertalot 1985, Bibliotheca Diatomologia, p. 62; pl.18, figs.22-23; pl.19, fig.1-10, pl.27, figs.1,4.

175. N. cryptotenelloides Lange-Bertalot 1993, Bibliotheca Diatomologica, p. 105; pl.50, figs.9-12; pl.51, figs.1-2.

176. N. erifuga Lange-Bertalot in Krammer \& Lange-Bertalot 1985, Bibliotheca Diatomologica, p. 69; pl.17, figs.10-12.

177. N. exilis Kütz. 1844, Die kieselschaligen Bacillarien oder Diatomeen p. 95; pl.4, fig.6.

178. N. germainii Wallace 1960, Notulae Naturae, pl.2, fig.1A-C.

179. N. hofmanniae Lange-Bertalot 1993, Bibliotheca Diatomologica, p. 116; pl.48, figs.12-18; pl.49, figs.1,2

180. N. irmengardis Lange-Bertalot in Lange-Bertalot \& Metzeltin 1996, Iconographia Diatomologica, p. 78; pl.80, figs.18-23.

181. N. notha Wallace 1960, Notulae Naturae, p.4; pl.1,figs.4A D.

182. N. phylleptosoma Lange-Bertalot in Lange-Bertalot \& Genkal 1999, Iconographia Diatomologica, p. 69; pl.13, figs.1-5.

183. N. radiosa Kutz. 1844, Die kieselschaligen Bacillarien oder Diatomeen, p. 91, fig. 4: 23.

184. Navicula radiosafallax Lange-Bertalot 1993, Bibliotheca Diatomologica, p. 131; pl.52, figs.1-3.

185. N. radiosiola Lange-Bertalot 1993, Bibliotheca Diatomologica, p. 132; pl.53, figs.4-8.

186. N. reichardtiana Lange-Bertalot in Lange-Bertalot \& Krammer 1989, Bibliotheca Diatomologica, p. 163, figs.98:19-27.

187. N. rostellata Kütz. 1844, Die kieselschaligen Bacillarien oder Diatomeen, p. 95, pl. 3, fig. 65.

188. N. schroeteri Meister 1932, Kieselalgen aus Asien, p. 38 fig. 100 .

189. N. sp. 4 Lange-Bertalot 2001, Diatoms of Europe 2, figs. 52: 8-29.

190. N. subrbynchocephala Hustedt 1935, Archiv für Hydrobiologie Supplement, p. 156; pl.1, fig.11.

191. N. symmetrica Patrick 1944, Boletim do Museu Naciona, nova serie, botanica, p. 5, fig.6.

192. N. trivialis Lange-Bertalot 1980, Cryptogamie: Algologie, $\mathrm{p}$ 31; pl.1, figs.5-9; pl.9, figs.1-2.

193. N. veneta Kütz. 1844, Die kieselschaligen Bacillarien ode Diatomeen, p. 95; pl. 30, fig. 76.

194. N. viridula (Kütz.) Ehren. 1838, Infusionsthierchen als vollkommene Organismen p. 183.

195. N. viridulacalcis Lange-Bertalot in Rumrich et al., 2000, Iconographia Diatomologica, p. 174; pl.38, fig.5.

\section{Navicula sensu lato}

196. Navicula cf. kotschyi; cf. grimmei Metzeltin et al., 2005, Iconographia Diatomologica, p. 60, figs. 14-19.

197. N. mitigata Hustedt 1966, Die Kieselalgen Deutschlands, (Figs. 62: 12).

198. N. seminulum Gruonw 1860, Über neue oder ungenügend gekannte Algen, figs. 76: 30-36.

199. N. medioconvexa Hustedt 1961, Die Kieselalgen Deutschlands, Österreichs und der Schweiz unter Berücksichtigung der übrigen Länder Europas sowie der angrenzenden Meeresgebiete, figs. 70: 1-7.

200. N. stroemii Hustedt 1931, Akademische Verlagsgesellschaft, figs. 69: 1-10; 83: 3 .

\section{Adlafia}

201. Adlafia miniscula (Grun.) Lange-Bertalot 1999, Iconographia Diatomologica p. 6, pl. 32.
202. A. parabryophila (Lange-Bertalot) Lange-Bertalot 1999 , Bibliothica Diatomologica, p. 38, pl. 89.

\section{Aneumastus}

203. Aneumastus stroesei (Østrup) D.G.Mann in Round, Crawford \& D.G. Mann 1990, The Diatoms: Biology and morphology of the genera, p. 663.

204. A. tuscula (Ehrenb.) Mann \& Sticle in Round et al., 1990 The Diatoms: Biology and morphology of the genera $\mathrm{p}$. 663.

\section{Craticula}

205. Craticula ambigua (Ehren.) D.G.Mann in Round, Crawford \& Mann 1990, The Diatoms: Biology and morphology of the genera, p. 666.

206. C. buderi (Hustedt) Lange-Bert. 2000, Iconogrphia Diatomolologica, p. 9, pl. 101.

207. C. citrus (Krasske) E.Reichardt 1997, Diatom Res., p. 12, pl. 305.

208. C. halopannonica Lange-Bertalot 2001, Diatoms of Europa 2 , p. 113,218 ; pl.85, figs.1-6.

\section{Fallacia}

209. Fallacia meridionalis Metzeltin et al., 2005, Iconographia Diatomologica, p. 61, figs. 30-37.

210. Fallacia pygmaea (Kütz.) A.J.Stickle \& D.G.Mann in Round, Crawford \& Mann 1990, The Diatoms: Biology and morphology of the genera, p. 668.

\section{Diadesmis}

211. Diadesmis confervacea (Kütz.) Grun. in Van Heurck 1880 Synopsis des Diatomees de Belgique, figs. 75: 29-31.

212. D. platensis Metzeltin et al., 2005, Iconographia Diatomologica, p. 56, figs. 1-10.

\section{Geissleria}

213. Geissleria decussis (Hustedt) Lange-Bert., 1996, Iconographia Diatomologica 2, p. 65.

\section{Hippodonta}

214. Hippodonta subtilissima Lange-Bertalot, Metzeltin \& Witkowski 1996, Iconographia Diatomologica, p. 265; pl.3, fig.16-23.

215. Hippodonta sp. Lange-Bertalot, Witkowski \& Metzeltin, 1996.

\section{Luticola}

216. Luticola aff. goepperttiana order aff. obligata (?) Krammer 1999, Iconographia Diatomologica, figs. 62: 1-18.

217. L. mutica var. lanceolata (Frenguelli) M.Aboal 2003, Diat. Monogr., p. 315.

218. L. mutica fo. intermedia Hustedt 1921, Hedwigia, figs. 63: 16.

219. L. mutica (Kütz.) D.G.Mann in Round et al., 1990, The Diatoms: Biology and morphology of the genera, p. 670

220. L. muticopsis (Van Heurck) D.G.Mann 1990, The Diatoms: Biology and morphology of the genera, p. 671.

221. L. peguana (Grun.) D. G. Mann 1990, The Diatoms: Biology and morphology of the genera, p. 671 .

222. L saxophila (Bock) ex Hustedt 1966, D. G. Mann 1990 The Diatoms: Biology and morphology of the genera, p. 671.

223. L. cf. uruguansis Metzeltin et al., 2005, Iconographia Diatomologica, p. 83, figs. 1-4

\section{Placoneis}

224.Placoneis elegines W. Smith, Metzeltin et al., 2005 Iconographia Diatomologica, figs 75, 1-9; SEM-Figs. 75, 10

225. P. witkowskii Metzeltin et al., 2005, Iconographia Diatomologica, p. 71, figs. 8-15.

\section{Sellaphora}

226. Sellaphora americana (Ehrenb.) D. G. Mann var. americana Metzeltin et al., 2005, Iconographia Diatomologica, p. 63, figs. 1-2. 
227. S. bacilloides Hustedt, Metzeltin et al., 2005, Iconographia Diatomologica, p. 66, figs. 18-21.

228. S. densistriata Lange-Bert. 1996, Iconogr. Diatomol. 2, p. 102, pl. 25: fig. 9.

229. S. hustedtii Krasske 1923, Hedwigia, figs. 71: 22-24.

230. S. laevissima (Kütz.) D.G.Mann 1989, British Phycological Journal, p. 2.

231. S. mutatoides Lange-Bertalot \& Metzeltin, Metzeltin et al., 2005, Iconographia Diatomologica, p. 64, figs. 6-9

232. S. pupula (Kütz.) Mereschkovsky 1902, Annals and Magazine of Natural History Ser., p. 187; pl.4, fig.1-5.

\section{Neidium}

233. Neidium ampliatum (Ehren.) Krammer 1985, Bibliotheca Diatomologia, p. 101.

234. N. binodiforme Krammer 1985, Bibliotheca Diatomologia, figs. 100: 6-8.

235. N. herynicum Mayer 1917, Bacillariales aus der Umgegend von Wurzburg, figs. 103: 11-16.

236. Neidium sp. Pfitzer, 1871

\section{Pinnularia}

237. Pinnularia acrosphaeria Rabenhorst 1853, Die SüssisserDiatomaceen, p. 45; pl.6, fig.36.

238. P. borealis Ehren. 1843, Verbreitung und Einfluss des mikroskopischen Lebens in Süd- und Nord-Amerika, 420 (132); pl.1/2, fig.6; pl.4/1, fig.5.

239. P. braunii (Grun.) Cleve 1895, Synopsis of the naviculoid diatoms, p. 75

240. P. subrostrata (A.Cleve) Cleve-Euler 1955, Die Diatomeen von Schweden und Finnland, p. 58.

241. P. frauenbergiana var. caloneiopsis Lange-Bertalot \& Werum, 2004, Iconographia Diatomologica, p. 84 figs. 1-15.

242. P. stomatophora (Grun.) Cleve 1895, Synopsis of the naviculoid diatoms, p. 83.

\section{Scoliopleura}

243. Scoliopleura peisonis Grun. 1860, Über neue oder ungenügend gekannte Algen, figs. 148: 21-24.

\section{Stauroneis}

244. Stauroneis anceps Ehren. 1843, Verbreitung und Einfluss des mikroskopischen Lebens in Süd- und Nord-Amerika, p. 306 [18 in reprint], 422; pl. 2/1: fig. 18.

245. S. nobilis Schumann 1867, Synopsis of the British Diatomaeeae, figs. 87: 1-2.

Family Epithemiaceae Grun. 1860 is represented by genera Epithemia (1 species) and Rhopalodia (2 species).

\section{Epithemia}

246. Epithemia sp. Brébisson, 1844: 33.

\section{Rhopalodia}

247. Rhopalodia gibberula (Ehren.) Otto Müller 1895, Rhopalodia ein neues Genus der Bacillariaceen, p. 58

248. R. parallela (Grun.) O.Müller 1895, Rhopalodia ein neues Genus der Bacillariaceen, p. 64; pl.1, figs.13,14.

Family Bacillariaceae Ehrenb. 1840 is represented by genera Bacillaria (1 species), Denticula (1 species), Hantzschia (1 species) and Nitzschia (32 species).

\section{Bacillaria}

249. Bacillaria paxillifer(O.F.Müller) T.Marsson 1901 Diatomaceen von Neu-Vorpommern, p. 254.

\section{Denticula}

250. Denticula kuetringii Grun. 1862, Verh. Kais.-Königl. Zoo.Bot. Ges. Wien., p. 546, 548; pl.28/12, fig.27.

\section{Hantzschia}

251. Hantzschia amphioxys (Ehren.) Grun. in Cleve \& Grun. 1880, Beiträge zur kenntniss der arctischen Diatomeen, p. 103.

\section{Nitzschia}

252. Nitzschia acicularis (Kütz.) W.Smith 1853, A synopsis of the British Diatomaceae, p. 43, pl. 15: fig. 12.

253. N. acuminata (W. Smith) Grun. Metzeltin et al., 2005, Iconographia Diatomologica, p. 194, figs. 3-4.

254. N. acuta Peragallo \& Peragallo 1899, Diatomées marines de France et des districts maritimes voisins, p. 281; pl.72, fig.19.

255. N. amphibia Grun. 1862, Verh. Kais.-Königl. Zoo.-Bot. Ges. Wien., p. 574; pl.28/12, fig.2.

256. N. calida Grun. 1880, Beiträge zur Kenntnis der arktischen Diatomeen, p. 75.

257. N. capitellata Hustedt, Hustedt 1985, The Pennate Diatoms, fig. 792.

258. N. clausii Hantzsch 1860, Beiträge zur Kenntnis der arktischen Diatomeen, p. 40; pl.6, fig.7. Description in Grun. 1892, p.559, 573.

259. N. coarctata Grun. 1880, Beiträge zur Kenntnis der arktischen Diatomeen, p. 68.

260. N. communis Rabh. Hustedt 1930, Bacillariophyta, f. 798. A. S. Atl. T. 348, figs. 10-19.

261. N. compressa (Bailey) C.S.Boyer 1916, The Diatomaceae of Philadelphia and vicinity, p. 116; pl.39, fig.7.

262. N. debilis (Arnott) Grun. in Cleve \& Grun. 1880, eiträge zur kenntniss der arctischen Diatomeen, p. 68.

263. N. dissipata (Kütz.) Grun. 1862, Verh. Kais.-Königl. Zoo.Bot. Ges. Wien., p. 561.

264. N. fonticola (Grun.) Grun. in Van Heurck 1881, Synopsis des Diatomées de Belgique Atlas, pl. 69, figs.15-20.

265. N. frustulum (Kütz.) Grun. in Cleve \& Grun. 1880, eiträge zur kenntniss der arctischen Diatomeen, p. 98.

266. N. ganderheimensis Krasske, Hustedt 1985, The Pennate Diatoms, fig. 804.

267. N. gracilis Hantzsch 1860, Hedwigia, p. 40; pl.6, fig.8.

268. N. hantzschiana Rabenhorst 1860, Hedwigia p. 40; pl.6, fig.6.

269. N. bungarica Grun. 1862, Verh. Kais.-Königl. Zoo.-Bot. Ges. Wien., p. 568; pl.28/12, fig.31.

270. N. intermedia Hantzsch ex Cleve \& Grun. 1880, eiträge zur kenntniss der arctischen Diatomeen, p. 95.

271. N. levidensis (W.Smith) Grun. in van Heurck 1881, Synopsis des Diatomées de Belgique Atlas, pl. 57, fig. 15.

272. N. levidensis (W. Smith) Grun. var. victoriae Metzeltin et al., 2005, Iconographia Diatomologica, p. 198, figs. 2-3.

273. N. linearis (C.Agardh) W.Smith 1853, A synopsis of the British Diatomaceae, p. 39; pl. 13: fig. 110.

274. N. obtusa var. lata Hagelstein Metzeltin et al., 2005 , Iconographia Diatomologica, p. 200, figs. 1-4.

275. N. palea (Kütz.) W.Smith 1856, A synopsis of the British Diatomaceae, p. 89

276. N. punctata (W.Smith) Grun. 1878, Algen und Diatomaceen aus dem Kaspischen Meere, p. 117.

277. N. recta Hantzsch ex Rabenhorst 1862, Die Algen Europa's,No.1283.

278. N. sinuata var. tabellaria (Grun.) Grun. in van Heurck 1881 , Synopsis des Diatomées de Belgique Atlas, p. 176; pl.60, fig.12-13.

279. N. spec. cf. rostellata Hustedt, Metzeltin et al., 2005, Iconographia Diatomologica, p. 204, fig. 14.

280. N. tenuis W.Smith 1853, A synopsis of the British Diatomaceae, p. 40; pl.13, fig.111.

281. N. umbonata (Ehren.) Lange-Bertalot 1978, Nova Hedwigia, p. 648-650; pl.1,2,4.

282. N. woltereckoides Gandhi, Gandhi 1998, Fresh water Diatoms of Central Gujarat, p. III, fig. 175.

283. Nitzschia sp. Hassall, 1845: 435

Family Surirellaceae Kütz. 1844 is represented by one genus Surirella (9 species).

\section{Surirella}

284. Surirella angusta Kütz. 1844, Die Kieselschaligen Bacillarien oder Diatomeen, p. 61; pl.30, fig.52.

285. S. apiculata var. penduriformis Frenguelli, Metzeltin et al., 2005, Iconographia Diatomologica, p. 222, figs. 3-9.

286. S. striatula Turpin 1828, Mémoires du Musée d'Histoire Naturelle; 363, pl. 15: figs. 2-10. 
287. S. linearis W.Smith 1853, A synopsis of the British Diatomaceae, p. 31; pl.8, fig. 58.

288. S. minuta Brébisson in Kütz. 1849, Species algarum, p. 38

289. S. ovalis Brebisson, Hustedt 1930, Bacillariophyta, p. 441, f. 860

290. S. spendida (Ehrenb.) Kütz., Metzeltin et al., 2005, Iconographia Diatomologica, p. 227, figs. 1-4.

291. S. splendida (Ehrenb.) Kütz., Hustedt, 1930, Bacillariophyta, p. 437 , fig. 851 .

292. Surirella sp. Turpin, 1828: 363.

\section{Acknowledgement}

The academic support by the Heads, Department of Zoology, H.N.B. Garhwal University and University of Allahabad, is acknowledged.

\section{References}

1. Chadha A \& Pandey DC. Algal flora of Allahabad. Part IV A general account. Biblio. Phycol.1983; 66: 141-178.

2. Chadha A. Investigations on the Algal Flora of Allahabad. D. Phil. Thesis, University of Allahabad, Allahabad 1979.

3. Gupta S \& Agrawal SC. Suvival and motality of diatoms Navicula grimmei, and Nitzschia palea affected by some physical and chemical factors. Folia Microbiol. 2007; 52 (2): 127-134.

4. Jyoti Verma (2011). Lotic Diatom diversity patterns in mountain chains (North India). Lambert Academic Publishing, pp. 176. SBN-13: 978-3-8465-0822-0.
5. Nautival P., Singh K.R. \& Verma J. (2010). Diatom flora in the artificial habitat (Pond) of Allahabad. Proceedings of National Academy of Sciences, Section B, Vol., 80 Part III.

6. Pandey UC \& Pandey DC. Addition to the algal flora of Allahabad-IV-Diatoms. Phykos 1980a; 19(2): 153-160.

7. Pandey UC \& Pandey DC. Addition to the algal flora of Allahabad-IV-Diatoms. Phykos 1980b; 1(2): 153-159.

8. Pandey UC \& Pandey DC. Diatom flora of Allahabad-I Proceedings of Indian Academy of Sciences, 1980c; 46(2): 350-355.

9. Pandey UC, Tiwari GL \& Pandey DC. Diatom flora of Allahabad, India II. Bibliotheca Phycologica, 1983.

10. Singh CS. A systematic account of the fresh water diatoms of Uttar Pradesh-II. In: Proceeding National Institute of Science, India 1961; 31(B): 203-223.

11. Singh CS. A systematic account of the fresh water diatoms of Uttar Pradesh-I. In: Proceeding National Institute of Science, India 1962; 32(B): 233-241.

12. Singh CS. A systematic account of the fresh water diatoms of Uttar Pradesh-I. In: Proceeding Nat. Institute of Science, India 1963; 29(B): 622-631.

\section{Cite this article as:}

Jyoti Verma, Prateek Srivastava, Ambrina Sardar Khan and Anita Gopesh. Check list of diatom taxa from Vindhyan rivers in Central Highland region. Annals of Plant Sciences 6.11 (2017) pp. 1726-1731.

doi: http://dx.doi.org/10.21746/aps.2017.6.11.2

Source of support: Department of Zoology, H.N.B. Garhwal University \& University of Allahabad, India.

Conflict of interest: Nil 